\title{
A NOVEL APPROACH TO INTEGRATE MEASURING INSTRUMENTS ONTO SINGLE SoC
}

\author{
T. Esther Rani ${ }^{1}$ and Dr.Rameshawa Rao ${ }^{2}$ and Dr.M.Asha Rani ${ }^{3}$ \\ ${ }^{1}$ CVR College of Engineering, Department of ECE, Ibrahimpatan, R.R.District, A.P., India \\ Email: estherlawrenc@gmail.com \\ ${ }^{2}$ Osmania University, Department of ECE, Hyderabad, A.P., India \\ Email: rameshwar_rao@hotmail.com \\ ${ }^{3}$ JNTUniversity, Department of ECE, Hyderabad, A.P., India \\ Email: ashajntu1@yahoo.com
}

\begin{abstract}
In this paper a novel proposal of SoC (Systemon-Chip) design approach is presented for the measurement, analysis, storage and display of physical and electrical quantities. The proposed System will be interfaced with sensors to perform the function of transducers. The LCD screen provides the display of both analog and digital data. At the circuit design level, transmission gates are used along with bulk-Silicon, through which the system occupies $80 \%$ less area and offers $60 \%$ more performance with very high rate of accuracy. This is an integrated form of all measuring instruments, Analyzers and recorders etc. The size of the system is so compact that it is comparable to the size of palm top computers or cell phones and operates with low power and high speed.
\end{abstract}

Index Terms-SoC, measurement, analysis, LCD display, Low-power, Compact

\section{INTRODUCTION}

Instrumentation is a technology of measurement which serves not only science, but all branches of engineering, medicine and almost every human endeavor. The knowledge of any parameter largely depends on the measurement. The in-depth knowledge of any parameter can be easily understood by the use of measurement, and further modifications can also be obtained. Whatever may be the nature of application, intelligent selection and use of measuring equipment depends on a broad knowledge of what is available and how the performance of the equipment renders itself for the job to be performed. But there are some basic measurement techniques and devices that are useful and will continue to be widely used also. There is always a need for improvement and development of new fquipment to solve measurement problems.

The major problem encountered with any measuring instrument is the error. Therefore, it is obviously necessary to select the appropriate measuring instrument and measurement method which minimizes error. To avoid errors in any experimental work, careful planning, execution and evaluation of the experiment are essential. Instruments rarely respond instantaneously to changes in the measured variables. Instead, they exhibit slowness or sluggishness due to such things as mass, thermal capacitance, fluid capacitance or electric capacitance[2]. In addition to this, pure delay in time is often encountered where the instrument waits for some reaction to take place. Such industrial instruments are nearly always used for measuring quantities that fluctuate with time. Here, the proposed instrument has no large massive components to be considered in the construction of the device. So, error is almost minimized.

The dynamic and transient behavior of the instrument is as important as the static behavior. The dynamic behavior of an instrument is determined by subjecting its primary element or sensing element to some unknown and predetermined variations in the measured quantity [1]. The proposed system has good speed of response and fidelity. Lag and dynamic error are almost minimized. Accuracy, precision, resolution and sensitivity of this design will be very high as this particular design is being realized with the minimum size transistors which results in minimum size of the instrument operated by low power and offers high speed. All the potential divider blocks of the design for the range selection are built by using transmission gates. With the transmission gates $15 \mathrm{k} \Omega$ to $60 \mathrm{k} \Omega$ resistors are built using minimum size transistors. By operating the transistors in the resistive region, the resistance can be improved[6]. The power supply to different blocks of the system is by using a battery.

\section{A. Mixed-Signal Hardware Description Languages:}

Both Verilog-AMS and VHDL-AMS have been defined and simulators that support these languages are emerging. These languages are expected to have a big 
impact on the design of mixed signal systems because they provide a single language and a single simulator that are shared between analog, digital, and eventually system designers. It will be much easier to provide a single design flow that naturally supports analog, digital and mixed signal blocks, making it simpler for these designers to work together [3].

For example, blocks like ADCs, DACs, PLLs, $\Sigma \Delta$ converters, discrete time filters (switched capacitor), etc, are easily and very efficiently modeled using the analog event -driven features of the AMS languages.

\section{SOC DESIGN}

\section{A. Processor:}

The use of microprocessors as an integral part of measuring instruments has given rise to a whole new class of instruments, called intelligent instruments.

\section{B. Memory:}

The basic advantage of digital operation is the storage capability, the stored waveform can be repetitively read out, thus making transients appear repetitively and allowing their convenient display on the LCD screen.

\section{I/O Interfacing:}

The inclusion of interfacing and a microprocessor provides a complete system for information acquisition, analysis and output [4].

The system is provided with LCD to display the readings of voltage, current, pressure, resistance, capacitance and inductance by using multi meter circuit. The wave forms can also be displayed on the screen along with the operation that is selected from the keypad. A keypad is provided for the selection of internal device, to store the values, digits to be used on calculator, zoom operations of the signal displayed and the process of data.

\section{Measuring Instruments:}

Digital meters, on the other hand, offer high accuracy, have high input impedance and are smaller in size. They give an unambiguous reading at greater viewing distances. The output available is electrical (for interacting with external equipment), in addition to a visual readout.

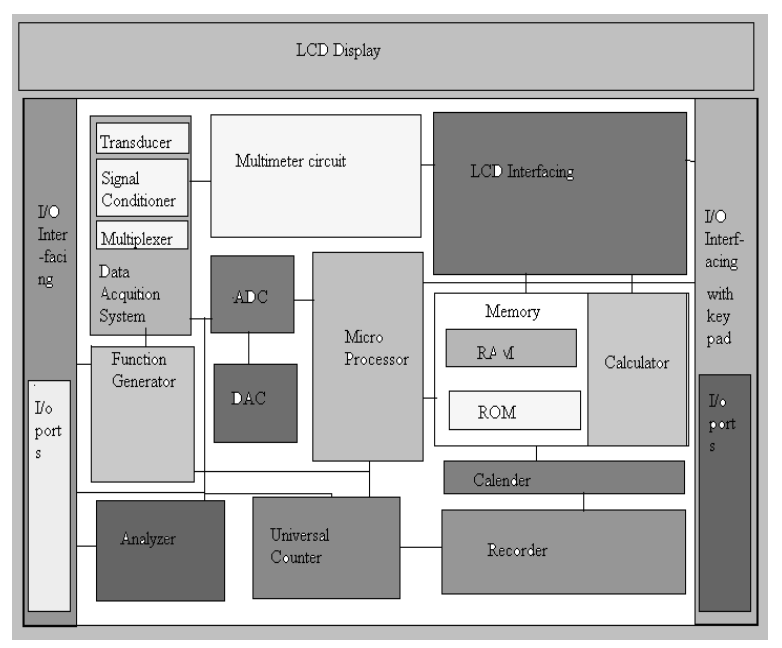

Fig 1: The proposed System architecture

\section{E. Passive Devices}

The passive devices like Resistor, Capacitor and Inductor can be placed on the chip.

An n-well resistor can be formed as the n-well resistivity may vary by several tens of percent with process. With typical sheet resistivities of about $1 \mathrm{k} \Omega / \square$, n-well resistors can prove useful where their absolute value is not critical. The metal layers available in CMOS technologies exhibit sheet Resistance on the order of $70 \mathrm{~m} \Omega / \square$ (for bottom layers) to $30 \mathrm{~m} \Omega / \square$ (for top layers). Thus, for resistor values common in analog design, metal layers are rarely used [5].

Capacitors prove indispensable in most of today's analog CMOS circuits. Several parameters of capacitors are critical in analog design. Non-linearity (voltage dependence), parasitic capacitance to the substrate, series resistance, and capacitance per unit are (density). In CMOS technologies modified for analog design, capacitors are fabricated as poly-diffusion, poly-poly, or poly-metal structures. The idea is to grow or deposit a relatively thin oxide between two floating conductive layers, thereby forming a dense capacitor with moderate bottom-plate parasitic (about 10 to 20\%) [5].

Challenges in industrial control and Instrumentation are autonomy \& co-ordination, accuracy, repeatability, intelligence, noise \& interference, high performance and wide voltage ranges. All the specifications can be met by employing different design methodologies for different blocks.

\section{F. Analogue to Digital Converter (ADC)}

All digital meters employ some kind of analog to digital (A/D) converters (often dual slope integrating type) and have a visible readout display at the converter output. The input signal is compared with an internally generated voltage which is increased insteps starting from zero. 


\section{G. Universal Counter}

All measurements of time period and frequency by various circuits can be assembled together to form one complete block, called a universal Counter timer.

\section{H. Function Generator}

A function generator produces different waveforms of adjustable frequency. The common output waveforms are the sine, square, triangular and saw tooth waves. The frequency may be adjusted, from a fraction of a Hertz to several hundred $\mathrm{kHz}$. The various outputs of the generator can be made available at the same time. Function generator consists of signal generator, amplifier or attenuator and filter.

\section{Wave Analyzers}

Wave analyzers are useful for measurement in audio frequency only. But, for measurements in RF range and above, special analyzers working on the principle of heterodyning are used which are called heterodyne wave analyzers. In this wave analyzer, the input signal to be analyzed is heterodyned with the signal from the internal tunable local oscillator in the mixer stage to produce a higher frequency. Spectrum analyzer provides a display of the frequency spectrum over a given frequency band. Spectrum analyzers use either a parallel filter bank or a swept frequency technique [7].

\section{J. Recording}

Recording is often carried out in order to perverse the details of measurement at a particular time. The information is fed directly to the processor for processing and control. Most of the critical parameters which influence the performance of the process or equipment have to be recorded for taking necessary action from time to time.

\section{TRANSDUCERS}

The sensing or detector element is that part of transducer which responds to a physical phenomenon. The transducer can be Resistive, Inductive, capacitive, electro-magnetic, Photo-emissive, photo-resistive, potentiometric, thermo-electric or Frequency generating [7]. Advantages in semiconductor technology have brought about high accurate, highly integrated miniaturized sensors that have transformed the way environmental and feed back control information is acquired and used in the industrial and instrumentation market.

\section{I/O PORTS}

The design quality of these circuits is a critical factor that determines the reliability, signal integrity, and inter chip communication in a system environment. Package is the first protection and I/O frame containing input output circuits is the second protection to the device. Any external hazards such as Electro Static Discharge (ESD) and noises should be filtered out before propagating to the internal circuits. As the system contains mixed signal design, i.e. some of the blocks like calculator, calendar, Processor and memory are completely digital and can be implemented using CMOS circuits. Function Generator, Analyzer and signal conditioner are the blocks which need to be implemented using analog signal design. So, for proper level shifting is to be provided for the good communication between the internal blocks of the design.

\section{LIQUID CRYSTAL DISPLAY (LCD)}

The purpose of liquid crystal display is to display both digital and analogue information. The rapid growth of electronic handling of numerical data has bought with it a great demand for simple systems to display the data in a readily understandable form. Display devices provide a visual display of numbers, letters, and symbols in response to electrical input, and serve as constituents of an electronic display system. The function of Oscilloscope is replaced by the LCD with the controls on the keypad provided.

\section{RESULTS}

The following blocks of proposed SoC are implemented by using Cadence tools in 0.18um Technology. The area and power of the corresponding blocks are listed in the table1 given below. The results are generated by Encounter ${ }^{\circledR}$ RTL compiler, with the technology library osu018_stdcells.

The processor of the system is considered as 16-bit so that it is useful for the data acquisition processing also [4]. Arithmetic and logic blocks of calculator are implemented in ALU. The selector switch is realized by using a 16x1 Multiplexer [2]. The LC oscillator designed for function generator is operating up to 3 $\mathrm{GHz}$ and consumes a $1874.4 \mathrm{pW}$ of power at $3.3 \mathrm{~V}$ power supply [5].

Table 1: Results of the blocks in the proposed system

\begin{tabular}{|l|l|l|l|l|}
\hline S.No & Block & $\begin{array}{l}\text { No. of } \\
\text { Stand } \\
\text { Cells }\end{array}$ & Area $\left(\mathrm{nm}^{2}\right)$ & $\begin{array}{l}\text { Total Power } \\
\text { (leakage+ } \\
\text { dynamic) (uw) }\end{array}$ \\
\hline 1. & CPU & 2176 & 74358 & 1212.603 \\
\hline 2. & ALU & 188 & 6628 & 61.749 \\
\hline 3. & $\begin{array}{l}\text { Selector } \\
\text { switch }\end{array}$ & 31 & 906 & 75.757 \\
\hline 4. & ADC & - & $23190 \times 10^{6}$ & 100 \\
\hline
\end{tabular}




\section{CONCLUSIONS \& Future SCOPE}

The system is flexible and capable of being expanded for future requirements, at the same time it is reliable and not has a down time greater than $0.1 \%$. The provided LCD to the device is monochrome. The interfacing can be done for color display. More number of transducers can be included in this system and the Data acquisition system design can be still more sophisticated.

\section{ACKNOWLEDGEMENT}

The authors would like to thank the management for providing the Cadence tools at Prof. Magdy A Bayoumi Centre for VLSI Design.

\section{REFERENCES}

[1] Doeblin, Measurement Systems_analysis, Mc Graw Hill.

[2] M.M. Mano, Digital Logic and_Computer Design, Prentice-Hall, India.

[3] www.analogdevices.com

[4] J.Uffenbech, The 8086/8088 Family:_Design, Programming and Interfacing, Prentice Hall, 1987.

[5] Behzad Razavi, Design of Analog CMOS Integrated Circuits, Tata Mc Graw Hill.

[6] Flavio Carbognani, Felix Buergin, Norbert Felber and Hubert Kaeslin, "Transmission Gates combined with level- restoring CMOS gates reduce_Glitches in lowpower, low-frequency Multipliers", IEEE Trans. Very Large Scale Integr (VLSI) syst, vol 16, No.7, pp 830836 , july2008.

[7] A.K.Sawhney and Puneet Sawhney, A Course in Electrical_and Electronic Measurements and Instrumentation, Dhanpat Rai \& Co (p) Ltd. 